\title{
Weak Zone Related Seismic Cycles in Progressive Failure Leading to Collapse in Brittle Crust
}

\author{
Chun an Tang, ${ }^{1,2}$ Mingli Huang ${ }^{2}$ and Xingdong ZhaO ${ }^{2}$
}

\begin{abstract}
Until quite recently our understanding of the basic mechanical process responsible for earthquakes and faulting was not well known. It can be argued that this was partly a consequence of the complex nature of fracture in crust and in part because evidence of brittle phenomena in the natural laboratory of the earth is often obliterated or obscured by other geological processes. While it is well understood that the spatial and temporal complexity of earthquakes and the fault structures emerge from geometrical and material built-in heterogeneities, one important open question is how the shearing becomes localized into a band of intense fractures. Here we address these questions through a numerical approach of a tectonic plate by considering rockmass heterogeneity both in microscopic scale and in mesoscopic scale. Numerical simulations of the progressive failure leading to collapse under long-range slow driving forces in the far-field show earthquake-like rupture behavior. En Echelon crack-arrays are reproduced in the numerical simulation. It is demonstrated that the underlying fracturing induced acoustic emissions (or seismic events) display self-organized criticality — from disorder to order. The seismic cycles and the geometric structures of the fracture faces, which are found greatly depending on the material heterogeneity (especially on the macroscopic scale), agree with that observed experimentally in real brittle materials. It is concluded that in order to predict a main shock, one must have extremely detailed knowledge on very minor features of the earth's crust far from the place where the earthquake originated. If correct, the model proposed here seemingly provides an explanation as to why earthquakes to date are not predicted so successfully. The reason is not that we do not understand earthquake mechanisms very well but that we still know little about our earth's crust.
\end{abstract}

Key words: Brittle failure, damage, seismicity, nonlinearity, heterogeneity.

\section{Introduction}

The recognition of a connection between earthquake related processes and critical phenomena, together with numerical models of earthquakes, have resulted in significant progress in the theoretical interpretation of earthquakes (BAK and TANG, 1989; Caldarelli et al., 1996; Cannelli et al., 1989; Heimpel, 1997; MiltenberGER et al., 1993; SCOTT, 1996; ZAPPERI, 1997). However, the mechanism behind this self-organization and the precise relationship between these largely different time

\footnotetext{
${ }^{1}$ LNM, Mechanics Institute, Chinese Academy of Science, Beijing, P.R.China, 100080

${ }^{2}$ CRISR, Northeastern University, Shenyang, Liaoning, P.R.China 110006 (current corresponding address)
} 
scales are still lacking (Miltenberger et al., 1993), and a clear picture of how earthquakes develop has yet to emerge (SAHIMI and ARBABI, 1996). Experimental investigations reveal that failure behavior of brittle material strongly depends on the material heterogeneity (MogI, 1985). One major question for numerical modeling of earthquake's is in what scale the heterogeneity should be represented in the model. Although from the viewpoint of micro-scale, many previous numerical models (CAldarelli et al., 1996; Miltenberger et al., 1993; SCOTt, 1996; ZAPPeri et al., 1997) are considered as representing a heterogeneity feature of the natural materials, most of the models behave homogeneously in macroscopic scale by exhibiting a failure process that is too brittle (SCHLANGEN and VAN MIER, 1994) and without revealing any seismic cycle behavior. However, seismicity is often not randomly distributed but spatially clustered (TANG and KAISER, 1998). These clusters were interpreted by Reifenberg (STEWART and SPOTTISWOODE, 1993) as areas of high local stress. We further propose that the spatial clustering can also be interpreted as areas of weakness even if in a low local stress level, which is believed to be related to the seismic cycles recorded in the crust failure. Pursuing the latter view, simulation is presented by using a Rock Failure Process Analysis code (TANG, 1997), RFPA ${ }^{2 D}$, developed based on finite element method (FEM) with proper consideration of failure mechanisms. In the simulation we try to specify the conditions of material heterogeneity both in microscale and in mesoscale.

\section{Model Setup and Numerical Results}

The geometry used for the simulation is a two-dimensional square block that is loaded in both spatial dimensions (as shown in Fig. 1). The model mesh contains $170 \times 170=28,900$ elements. We introduce the mesoscopic scale heterogeneity for some nonuniformly distributed weaker inclusions ( 5 weaker inclusions marked 1, 2, 5 with their strength and elastic modulus one half lower than that of the matrix are setup in the model). The mesoscopic heterogeneity represents the variation of the inclusion size, shape, mechanical properties and the volume percentage. The microscopic heterogeneity represents the variation of the mechanical properties for the elements composing the inclusions. Every inclusion has its own geometric and mechanical properties. The microscopic heterogeneity for the elements composing the inclusions and matrix is achieved to assign to the elements random strength and elastic modulus by assuming a Weibull distribution (TANG et al., 1993). We use a smeared failure approach that a microfracturing or quake occurs when the stress of a element satisfies a strength criterion. The simulation proceeds as follows. We apply an external displacement with a constant rate in the $y$ direction and a confining stress in the $x$ direction of the block. We compute the stress and deformation in each element. The external displacement in $y$ direction is then slowly increased step by step. At certain steps the stress in some elements satisfies 


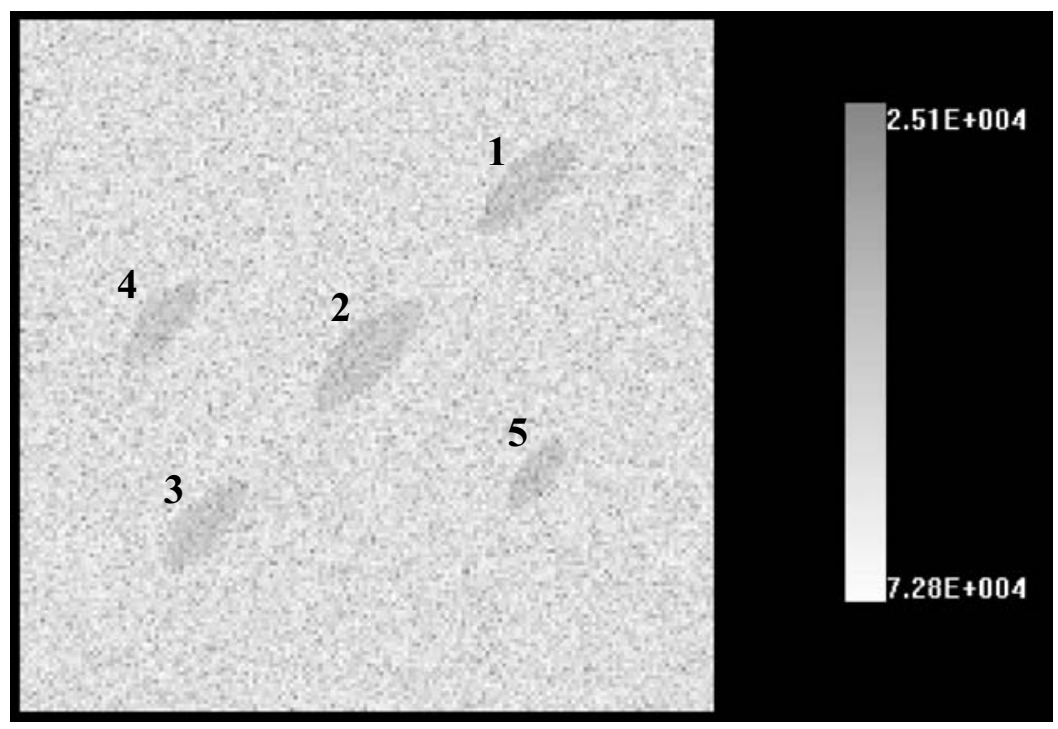

Figure 1

Numerical model mesh. There are five weak zones called the inclusion zones, located in the sample with the average elastic modulus (and strength) of the inclusions marked 1,3, 4, and 5 being 50\% lower than the surrounding materials, and the inclusion marked number 2 being $75 \%$ lower. The brightness of the gray shading indicates the heterogeneity of the element elastic modulus (high $=$ white, low $=$ black).

strength criterion, the element is damaged either in shear or in tension and becomes weak according to the rules specified above. The stress and deformation distribution throughout the block is then adjusted instantaneously after each rupture to reach the equilibrium state. At positions with increased stress due to stress redistribution, the stress may exceed the critical value and further ruptures are caused. The process is repeated until no failure elements are present. More external displacement is then applied. In this way the system develops a macroscopic fracture. Energy is stored in the elements during the loading process and is released as acoustic emissions through the onset of element failures. Owing to the stress redistribution and the long-range deformation induced interactions, a single element failure may induce an avalanche of additional failures in neighbor elements leading to a chain reaction releasing more energy.

The pattern of fracture process shown in Figure 2 reproduces the main features of mechanical tests on brittle materials and mimics the basic characteristics of earthquakes. As shown in Figure 2, the rock first develops isolated microfractures which are nucleated in the weak regions (stage B in step 68). As the applied strain increases, more weak regions develop fractures (stage $\mathrm{C}$ in step 86), while the other regions remain relatively intact. After some time the fractures coalesce (chain reaction) and a shear failure zone emerges that explains the existence of selforganized criticality (stage D in step 108). 


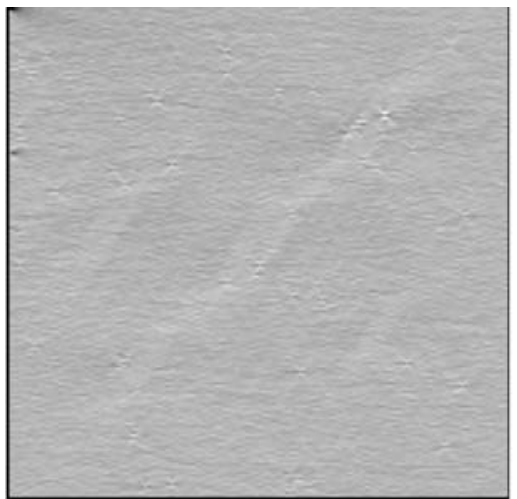

A-Step 25

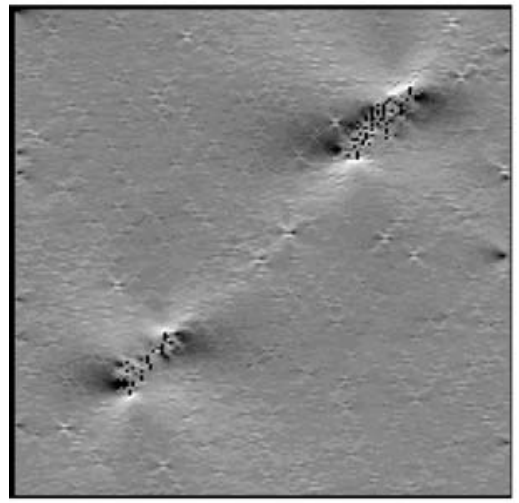

B-Step 68

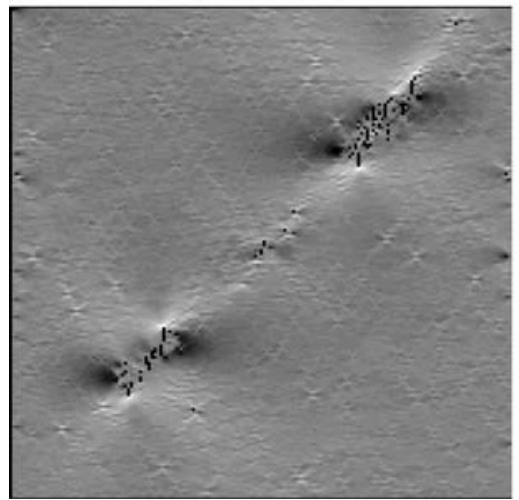

C-Step 86

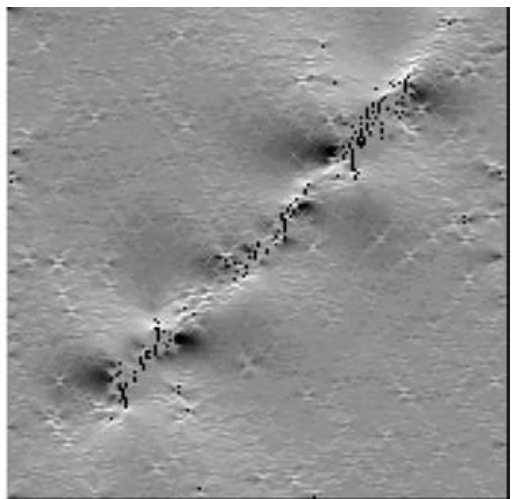

D-Step 108

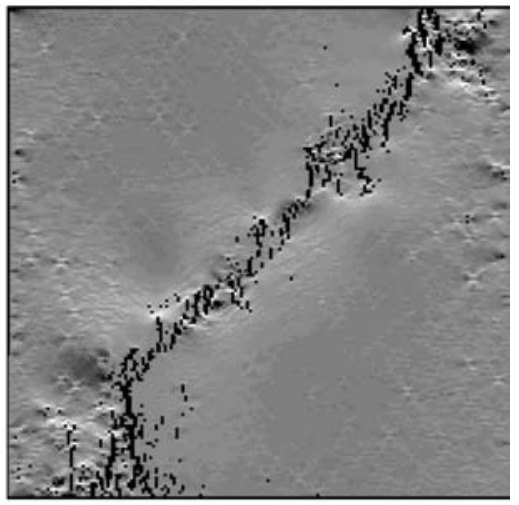

E-Step 122

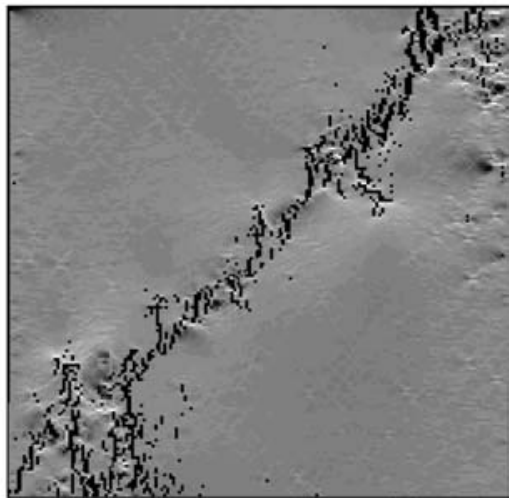

F-Step 149

Figure 2

Numerical simulation of failure mode (simulated with RFPA ${ }^{2 D}$ ) (Dark areas indicate zones of lower stress relative to the rock strength). 


\section{Discussion and Conclusion}

The progressive failure of brittle materials has been exhaustibly investigated by numerical studies using lattice network models (CALDARElli et al., 1996; MiLteNBERGER et al., 1993), or granular models (SCOTT, 1996; LIU et al., 1994), or even an electrical resistor network (ZAPPERI, 1997) as a analogy. The results from our failure simulation show other features.

Firstly, a Coulomb criterion envelope with a tensile cut-off (BRADY and BROWN, 1993 ) is used so that the failure of the elements may be either in shear or in tension depending on the combination of the principal stresses $\sigma_{1}$ and $\sigma_{2}$ for the twodimensional model. However, most of the existing numerical models are difficult to simulate the complex failure modes involving mode I and mode II fractures in heterogeneous geomaterials. By introducing threshold value for the length of a spring without considering the difference between tensile strength and compressive (or shear) strength, the predicated damaged bands in most of the lattice network models (ZAPPERI, 1997; MARDER and LiU, 1993; ABrAham et al., 1994) can only resemble the geometrical structure of the failure surface in tensile fracture mode. However, instead of modeling the failure process in a microscopic scale (such as atom model), the numerical models for geo-materials are in a mesoscopic scale, and, in which scale, rock exhibits a strength difference between tension and compression due to the existence of defects or flaws. Near the earth's surface, overburden pressures are small so that a large-scale tensile failure phenomenon may occur. At seismogenic depths, however, unless fluid pressures are abnormally high, all principal stresses become compressive and the rock must fail mainly in shear or in the combination of shear and tension (LOCKNER and MOORE, 1992). While tensile fracture is a relatively simple failure mode, shear failure of brittle rock can be a more complicated process, involving a number of stages of damage accumulation (LOCKNER and MOORE, 1992), which represents a great challenge to numerical modelers in geophysics. Scott's granular model (SCOTT, 1996) appears to offer a possible shear mechanism for the occurrence of earthquakes, but whether such a granular model is a good representation of the brittle crust is also questioned (SCHOLZ, 1996).

The failure mode shown in Figure 2 can explain the formation of fault which is nucleated in relatively intact rock and tends to grow and organize, not as straight initiated, but more often as self-affine structures composed of many strands of varying lengths, leading to the geological concepts of fault segments and barriers. It is of interest to compare the numerical results with the experimental observations made by LAJTAI et al. (1994). After examining the failure mode of potash salt rock both in site and in laboratory, LAITAI et al. defined two types of fracture patterns: en echelon tensile crack-array pattern and en echelon shear crack-array pattern. A further examination of the numerical results shown in Figure 2 suggests that the simulation agrees well with the experimental observation made by LAITAI et al. As shown in the figures, during the pre-peak failure stage, the fracture of the model sample involves the 
propagation of numerous tensile cracks that normally run parallel to the direction of the axially compressive stress. The weak inclusions more or less control the crack path at the mesoscopic scale (stage B-D). At the macroscopic scale of the model, the loading stress controls the fracture propagation direction. In the post-peak failure stage or the strain-weakening stage (stage D-F) we can see the appearance of inclined (so-called shear) fracture zone in macroscopic scale. Eventually, the whole en echelon array collapses forming a so-called shear fracture or fault. It was found that due to the stress redistribution, the area away from the shear zone was elastically restored.

Secondly, it is noted that the existence of such weak zones demonstrates a more realistic image of earthquake sequences. The simulation shown in Figure 3 illustrates the associated seismicities in the evolution of a fault zone, and presents a complete image of foreshocks, main shock and aftershocks. Due to the mesoscopic inhomogeneities, the fracture development occurred in the 1st week inclusion until the much stronger neighborhood matrix is encountered. Then, fracture growth stops and another fracture nucleates in other weak zones (3rd weak inclusion). The growth of the new fractures also stops when it encounters another stronger area between the weak inclusions, and so on. This gives rise to large fluctuations in the cumulative elastic energy released as seismic events during fracture. This is repeated in cycles that are similar to the earthquake data (see the marks A, B, ..F shown in Fig 4). It is found that only three weak inclusions (marked 1,2,3) along a line (the potential shear zone) are active. Thus we may view seismicity in a region as a sequence of fracturing cycles, where each cycle represents a progressive cooperative stress buildup and fracture nucleation that culminate in some manner of a critical point which is characterized by global failure in the form of a large earthquake. Note that a similar conclusion has been inferred by SAHIMI and ARBABI (1996).

Understanding the factors that control foreshock occurrence is critical for determining how large earthquakes initiate and whether reliable short-term prediction will ever be possible (ABERCROMBIE and Mori, 1996). The seismicity illustrated in Figure 4 shows more clearly how the microfracturing evolves in the manner forming the entire imaging of foreshocks, main shock and aftershocks. The seismic events (fracture-avalanche statistics) and their energy lost during failure are computed by recording the number of failed elements and the size of an avalanche. Before the main shock occurs, long-range correlation develops at many scales that leads to the cascade of events (earthquakes) at an increasingly larger scale. Note the irregularity of the events in Figure 4. At several points the shock is almost dying, and its continued evolution depends on minor details of the material properties in the model. Similar seismic features have been observed in experiments investigating quasi-static fault growth on Westerly granite and Berea sandstone (LOCKNER et al., 1991; LoCKNer and MoOre, 1992). These results imply that self-similarity and criticality on a fault emerge during an earthquake cycle, and suggest that the character of local seismicity can be useful in earthquake forecasting by revealing how advanced a fault is within its cycle (HEIMPEL, 1997). Thus in order to predict a main 


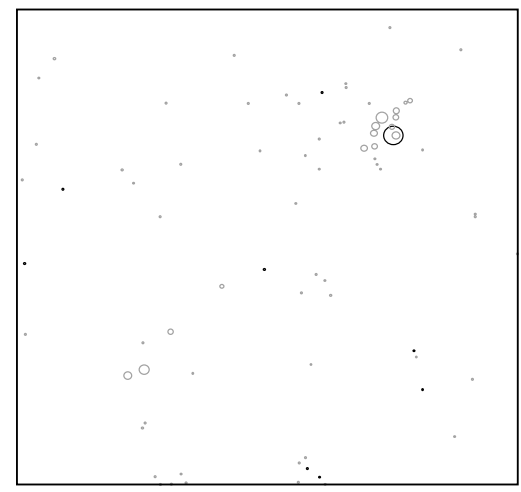

A-Step 25

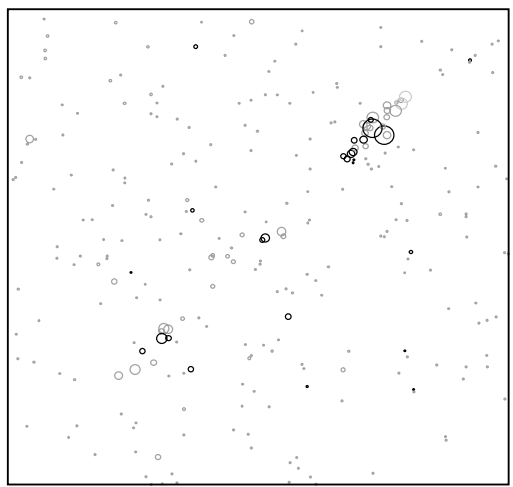

B-Step 68

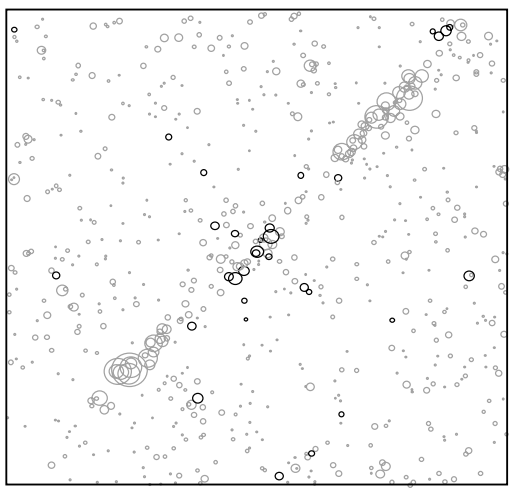

C-Step 86

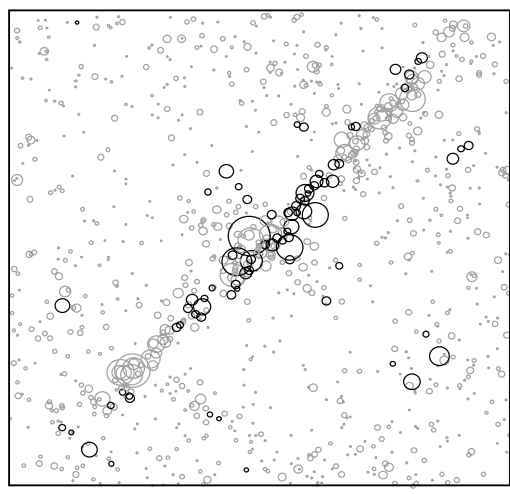

D-Step 108

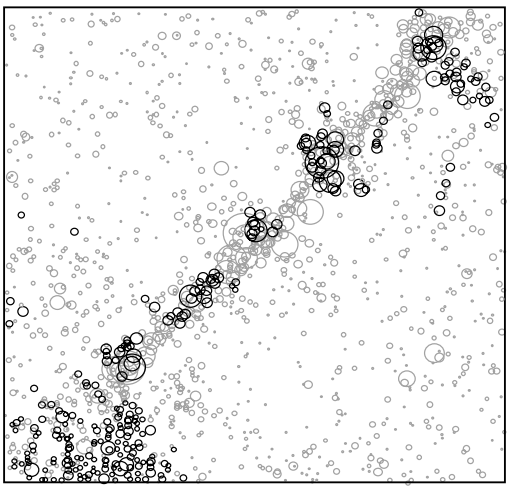

E-Step 122

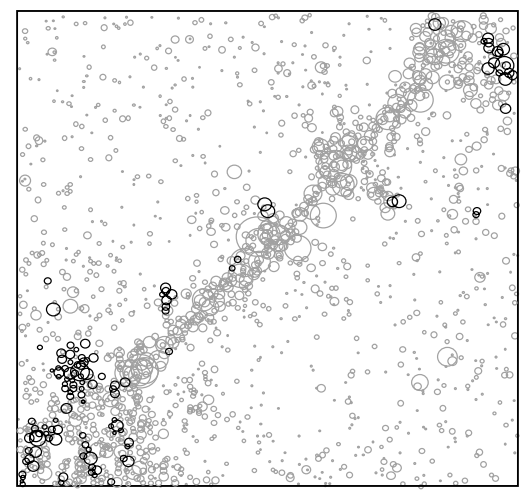

F-Step 149

Figure 3

Numerical simulation of source location of seismicity (simulated with RFPA ${ }^{2 \mathrm{D}}$ ) (black circles indicate event locations during current loading step; gray circles represent cumulative events of previous steps). 

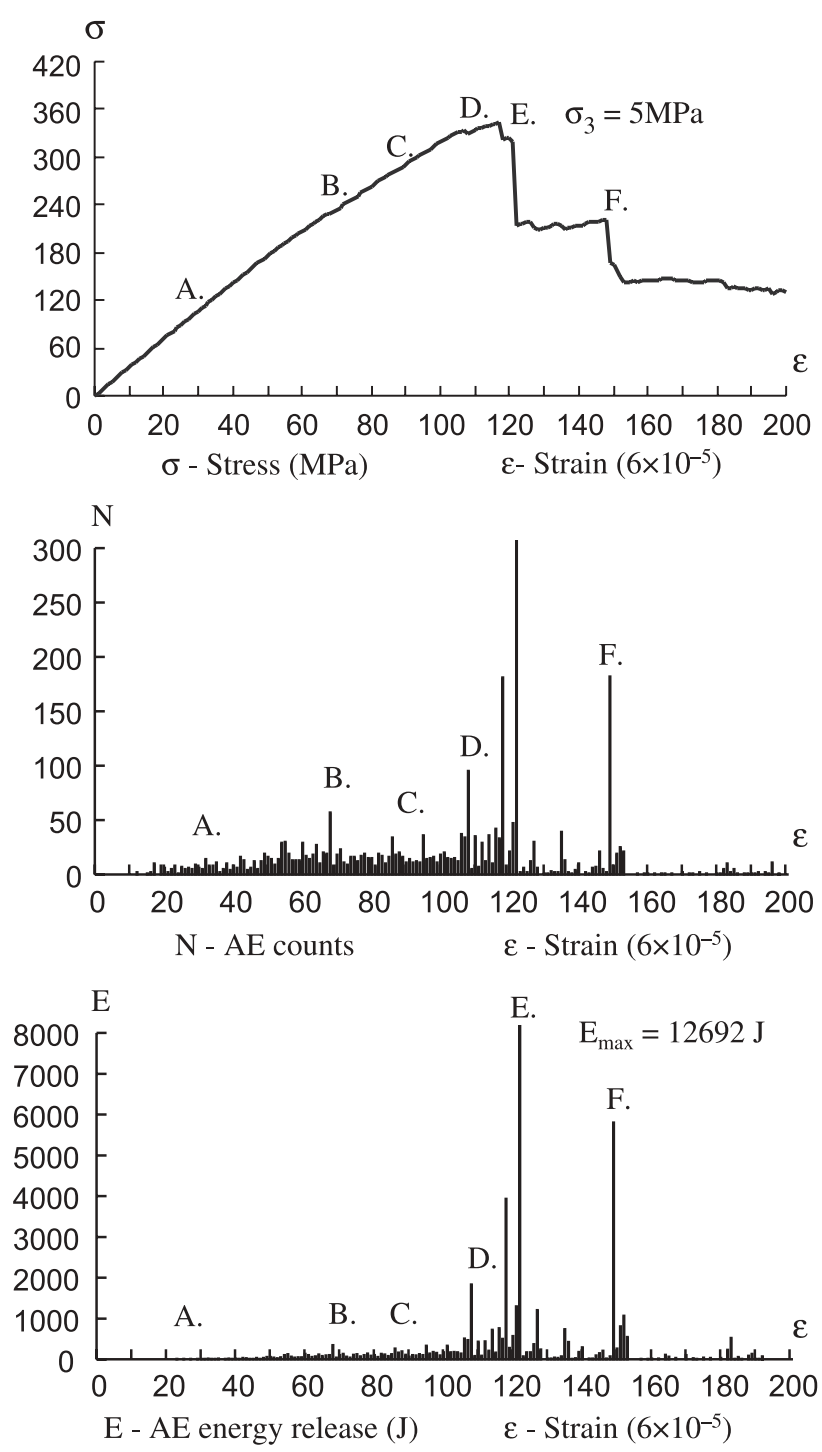

Figure 4

Stress-stain curve, AE counts energy release (simulated with RFPA $^{2 \mathrm{D}}$ ).

shock, one must have extremely detailed knowledge of very minor features of the earth's crust far from the place where the earthquake originated. If correct, the model proposed here seems to provide an explanation as to why earthquakes thus far are not predicted so successfully, and the reason is not that we inadequately understand earthquake mechanisms but that we still know little about our earth's crust.

The increase of the external inputted energy is balanced by the damage in such a way that the stress drops in a brittle manner. Figure 4 also illustrated that every large 
increase of acoustic emissions and released elastic energy resulted in a large stress drop (see the curves with marks D, E and F).

If we define the avalanche size $n$ as the number of elements damaged for a given magnitude increment, and find that, as in the lattice model (MARDER and LIU, 1993; ZAPPERI, 1997), the frequency and magnitude relation in our simulation also exhibits a power-law behavior (TANG, 1997).

The point that the weakening of large faults by elevated pore-fluid pressure is suggested as one solution to the San Andreas Fault heat-flow paradox is questioned by SCOTT (1996) with his granular model. Again, our model also removes the need for this relatively complex idea. Although there are many factors that may influence the initiation of earthquakes, factors such as pore-fluid pressure, rate-dependent friction or slip-dependent strength, etc., are not the prerequisites to initiate an earthquake. In the self-organization criticality (SOC) systems (BAK and TANG, 1989), instability occurs when the value of the relevant field exceeds locally some critical value and there is no parameter (pore-fluid pressure, for instance) to be adjusted in order to reach criticality - the system self-organizes into a critical state (CALDARELLI, 1996).

We note that the present model can also be used to simulate the failure process and organization of slow earthquake (SEGALL, 1996) (swarms) and explain why failure sometimes occurs slowly and at other times catastrophically by adjusting the heterogeneity and stiffness in the model respectively (KAISER and TANG, 1998; TANG et al, 1993). It is worth noting that instead of a constant strain rate, when we load the model in a constant stress rate, the failure process will be put into a sweeping of instability. This is quite similar to the reported result with a resistor network model (ZAPPERI, 1997), in which case if the system is driven by imposing a constant current, no steady-state is observed and the system is driven to an instability corresponding to the critical current of the voltage-driven experiment. These simulations should eventually improve our understanding and help to determine under which conditions the failure process will result in stable faulting that if takes a long time to complete (SEGALL, 1996) or in unstable faulting which produces an earthquake.

\section{Acknowledgement}

The authors are grateful for the support of this work from the Chinese National Key Fundamental Research 973 Programme (G1998040700) and the Natural Science Foundation of China (No.49974009 and 50174013).

\section{REFERENCES}

Abercrombie, R.E. and Mori, J. (1996), Occurrence Patterns of Foreshocks to Large Earthquakes in the Western United States, Nature 381, 303-307. 
Abraham, F.F., Brodbeck, D., Rafey, R.A., and Rudge, W.E. (1994), Instability Dynamics of Fracture: A Computer Simulation Investigation, Phys. Rev. Lett. 73, 272-276.

BAK, P. and TANG, C. (1989), Earthquakes as a Self-organized Critical Phenomenon, J. Geophys. Res. 94 , $15,635-15,637$.

Brady, B.H.G. and Brown, E.T. Rock Mechanics (second edition), (Chapman and Hall London, 1993).

Caldarelli, G., Tolla, F.D.D., and Petri, A. (1996), Self-organization and Annealed Disorder in a Fracturing Process, Phys. Rev. Lett. 77, 2503-2506.

Cannelli, G., Cantelli, R., and Cordero, F. (1989), Self-organized Criticity of the Fracture Processes Associated with Hydrogen Precipitation in Niobium by Acoustic Emission, Phys. Rev. Lett. 70, 3923-3925.

Heimpel, M. (1997), Critical Behavior and the Evolution of Fault Strength During Earthquake Cycles, Nature 388, 865-868.

KAISER, P.K. and TANG, C.A. (1998), Numerical Simulation of Cumulative Damage and Seismic Energy Release in Unstable Failure of Brittle Rock-Part II. RIB Pillar Collapse, Int. J. Rock Mech. Min. Sci. 35(2), 123-134.

Lajtai, E.Z., Carter, B.J., and Duncan, E.J.S. (1994), En echelon Crack-Arrays in Potash Salt Rock, Rock Mechan. and Rock Engin. 27(2), 89-111.

LiU, Z., Myer, L.R., and CoOK, N.G.W., Numerical simulation of the effects of heterogeneities on macrobehavior of granular materials, In Computer Methods and Advances in Geomechanics, (Siriwardane and Zaman, eds.) (Balkema, Rotterdam, 1994) pp. 611-616.

Lockner, D.A., Byerlee, J.D., Kuksenko, V., Ponomarev, A., and Sidorin, A. (1991), Quasi-static Fault Growth and Shear Fracture Energy in Granite, Nature 350, 39-42.

Lockner, D.A. and Moore, D.E., Microcrack interaction leading to shear fracture, Rock Mechanics (Tillerson and Wawersik, eds.) (Balkema, Rotterdam, 1992) 807-814.

Marder, M. and Liu, X.M. (1993), Instability in Lattice Fracture, Phys. Rev. Lett. 71, 2414-2420.

Miltenberger, P., Sornette, D., and Vanneste, C. (1993), Fault Self-organization as Optimal Random Paths Selected by Critical Spatiotemporal Dynamics of Earthquakes, Phys. Rev. Lett. 71, 3604-3607.

Mogi, K. Earthquake Predication (Academic Press, Harcourt Brace Jovanovich, Tokyo 1985).

SAhimi, M. and Arвabi, S. (1996), Scaling Laws for Fracture of Heterogeneous Materials and Rock, Phys. Rev. Lett. 77, 3689-3692.

Segall, P. (1996), Earthquakes: Slow Down for Safety, Nature 383, 15-22.

SChlangen, E. and VAN Mier, J.G.W., Fracture simulations in concrete and rock using a random lattice. In Computer Methods and Advances in Geomechanics, (Siriwardane H.J. and Zaman M.M., eds) (Balkema, Rotterdam, 1994) 1341-1346.

Scholz, C.H. (1996), Faults without Friction? Nature 381, 556-557.

ScotT, D.R. (1996), Seismicity and Stress Rotation in a Granular Model of Brittle Crust, Nature 381, $592-595$.

Stewart, R.D. and Spottiswoode, S.M., A technique for determing the seismic risk in deep-level mining. In Rockbursts and Seismicity in Mines (Young, ed.) (Balkema, Rotterdam, 1993) 153-158.

TAng, C.A., Hudson, J.A., and Xu, X.H. (1993), Rock Failure Instability and Related Aspects of Earthquake Mechanisms, China Coal Industry Publishing House, Beijing.

TANG, C.A. (1997), Numerical Simulation of Progressive Rock Failure and Associated Seismicity, Int. J. Rock Mech. Min. Sci. 34, 249-262.

TANG, C.A. and KAISER, P.K. (1998), Numerical Simulation of Cumulative Damage and Seismic Energy Release in Unstable Failure of Brittle Rock-Part I. Fundamentals, Int. J. Rock Mech. Min. Sci. 35, 113-121.

Zapperi, S., Vespignani, A., and StAnley, H.E. (1997), Plasticity and Avalanche Behavior in Microfracturing Phenomena, Nature 388, 658-660.

(Received August 8, 2000, accepted April 25, 2002)

$$
\begin{aligned}
& \text { (2) To access this journal online: } \\
& \text { (ID) http://www.birkhauser.ch }
\end{aligned}
$$

